\title{
Téoros
}

Revue de recherche en tourisme

\section{Les évolutions récentes de l'économie hôtelière}

\section{Gérard Guibilato}

Volume 7, numéro 3, novembre 1988

Économie du tourisme

URI : https://id.erudit.org/iderudit/1080382ar

DOI : https://doi.org/10.7202/1080382ar

Aller au sommaire du numéro

Éditeur(s)

Université du Québec à Montréal

ISSN

0712-8657 (imprimé)

1923-2705 (numérique)

Découvrir la revue

Citer cet article

Guibilato, G. (1988). Les évolutions récentes de l'économie hôtelière. Téoros, 7(3), 29-35. https://doi.org/10.7202/1080382ar d'utilisation que vous pouvez consulter en ligne.

https://apropos.erudit.org/fr/usagers/politique-dutilisation/ 


\section{Les évolutions récentes

Gérard Guibilato*

Le secteur hôtelier a considérablement évolué depuis vingt ans dans ses structures comme dans son activité et sa technicité. C"est un secteur économique avec de fortes traditions, arrivé à maturité, mais qui est le terrain d"innovations importantes dans divers domaines. Ses performances économiques et financières récentes font état de quelques difficultés inégalement ressenties selon la zone géographique et le type d'entreprises considérés. Nous nous proposons donc dans le présent article de faire le point sur ces évolutions et ces performances, en analysant tour à tour les changements intervenus dans les structures de l'offre et son mode de fonctionnement, puis dans ses caractéristiques économiques.

\section{Les tendances récentes sur le marché hôtelier international}

L'observation de l'évolution récente des marchés hóteliers à travers le monde laisse apparaitre un certain nombre de tendances que l'on peut résumer principalement à:

- un développement rapide des chaines hôtelières en données absolues et en parts de marché, ceci dans un contexte d'expansion générale de l'offre hôtelière.

- ce développement des chaines procède à la fois d'une internationalisation croissante et d'une segmentation plus affinée des marchés.

- la croissance du marché hớtelier et des chaines s'accompagne également de phénomènes de concentration économique et financière sous diverses formes.

- une technicité accrue en matière de management, avec une sophistication des techniques à différents niveaux et dans divers domaines (construction, production, commercialisation, gestion).

Nous allons examiner plus en détail chacune de ces tendances.

\footnotetext{
- Directeur de l'École Superieure des Sciences Economiques et Commerciales/Cornell School of Hotel Ad. ministration, Paris,
}

\section{Le développement tous azimuths des chaînes hôtelières}

Au cours des dix dernières années, un certain nombre de facteurs ont contribué à accroitre l'offre hôtelière:

- changements démographiques: impact du baby-boom, nombre accru de retraités.

- augmentation du nombre de familles avec deux salaires et des revenus en général.

- développement des échanges et croissance économique attendue des années 80 .

- incitations fiscales et financières à construire des hôtels.

- impact de l'inflation et spéculation sur les tendances inflationnistes.

- disponibilité de capitaux venant de nouveaux investisseurs.

- limitation voire absence de participation en capital et d'investissements directs des compagnies hôtelières.

C'est évidemment devenu un lieu commun d'affirmer que le développement quantitatif et qualitatif de l'offre hótelière mondiale s'est effectué par le biais de la croissance des chaînes intégrées.

Selon une étude menće par Hotels and Restaurants International", le nombre de chambres appartenant aux vingt premiers groupes hôteliers intégrés mondiaux a augmenté de $149 \%$ entre 1970 et 1985 , passant de 576000 à 1423000 .

Certes, la majorité de l'offre hồtelière reste encore constituée d'unités indépendantes, mais les chaînes intégrées érodent progressivement la part des indépendants ou des groupements volontaires, car leur progression est supérieure à l'accroissement moyen du parc hôtelier.

Ainsi par exemple, en France, la part des chaines intégrées dans l'hôtellerie homologuée (en nombre de chambres) est passée de $14,8 \%$ en 1986 à $17,7 \%$ en 1987 , avec 89000 chambres sur un total de 507000 . En Grande-Bretagne, en 1987, les chaines représentaient $11 \%$ du nombre d'hótels mais $30 \%$ des chambres.

Cette croissance quantitative induit également des modifications qualitatives du parc. Les chaines proposent souvent un produit hôtelier standardisé, conforme aux attentes préalablement définies des marchés visés.
Ceci leur permet notamment de sécuriser leurs clients en rendant leur offre consistante à travers les différentes localisations. Dans la mesure où la majorité de ces produits hơteliers sont de création relativement récente, la qualité de ce parc est supérieure à la moyenne.

En outre, la taille moyenne des établissements de chaînes est plus élevée, favorisant une gestion et une commercialisation plus indusirialisée des unités. En France, la capacité moyenne des hôttels indépendants est de 22 chambres par établissement, contre 80 pour les établissements de chaine intégrées.

Sous la pression des chaines intégrées, de nombreux indépendants se regroupent à présent dans des chaines volontaires ou groupements hôtteliers, assurant des services communs aux adherents, notamment au plan commercial, et des économies d'échelle par centrale d'achat. Certaines de ces chaines atteignent une dimension internationale, comme Best Western ou Relais et Chateaux.

\section{Une internationalisation accrue}

Le développement des groupes intégrés. s'effectue par la croissance sur leurs marchés nationaux respectifs mais également vers l'étranger. Au départ, cette internationalisation fut le fait de chaînes américaines: Holiday Inns, Sheraton, Hilton, Marriott furent longtemps les seules sociétés hôtelières transnationales importantes. À présent, des groupes transnationaux battant d'autres pavillons émergent parmi les grands de l'hótellerie internationale: Trust House Forte, Grand Metropolitan, Ladbroke (G.B.), Accor, Pullman, Meridien (France), Nikko, Ana (Japon), Four Seasons (Canada) (cf. tableau 1).

L'expansion internationale des chaines s'inscrit dans une stratégie de croissance à outrance de la plupart de ces groupes. Leurs marchés nationaux couverts, voire saturés, ils se tournent tout naturellement vers des marchés étrangers plus ouverts, offrant des localisations attractives soit qu'il s'agisse de nouvelles destinations touristiques, soit de centres d'affaires en développement.

L'internationalisation procède également $\mathrm{d}^{+} u n e$ stratégie de fidélisation de la clientèle. les chaînes cherchant ainsi à accompagner leurs clients dans leurs déplacements à l'étranger. Cette tendance se trouve encore renforcée par la création de systèmes de 
TABLEAU 1

Deux groupes transnationaux: Accor et Pullman $/$. H

Alocor

Repartion da Parc Hoelier au 31.12.87; 713 hotels, 84786 chambies

Par marque

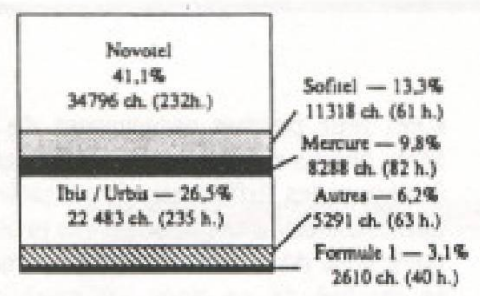

Par acoe gobgraphique

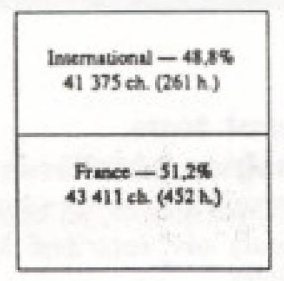

Repartition du chiffre d' alfaire en 1987: 72276 MF.T.T.C.

Par marque

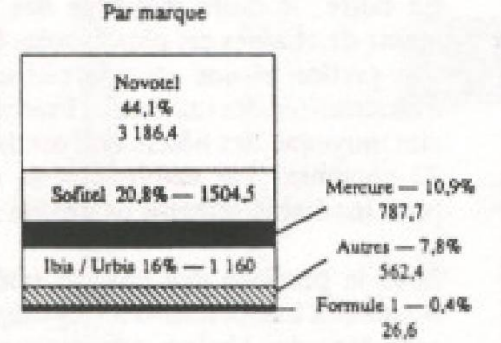

Par zone gtographique

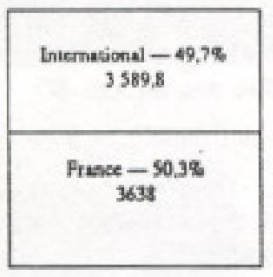

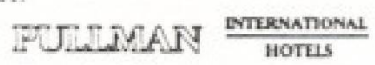

Repartion đu Parc Hótelię au 31.12.87: 212 bovels. 27554 chambres

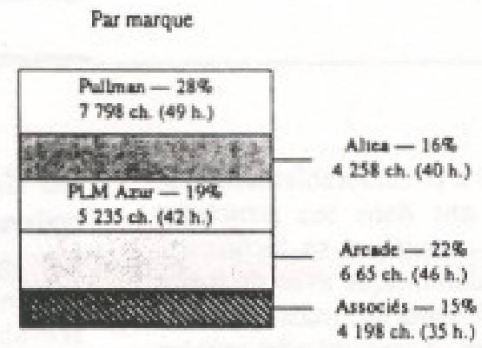

Par zone geographique

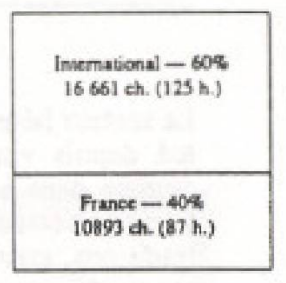

Repartition du chiffre d'affaire en 1987: 2 in M.F.T.T.C. *
Par marque

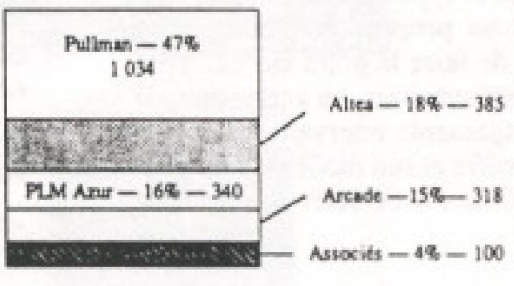

Far zone geographique

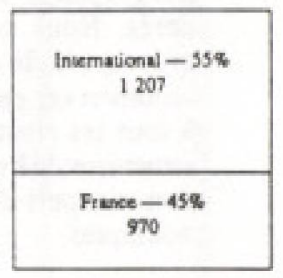

- Han irackimi réservation internationaux propres à chaque groupe ou communs à plusieurs compagnies.

Plus encore, pour les chaines de haut de gamme, l'implantation dans telle ou telle métropole devient un élément de prestige, voire une obligation dans une stratégie d'image de marque.

Un avantage supplémentaire d'une diversification géographique accrue réside dans une bonne répartition des risques, eu égard notamment aux problèmes de fréquentation et aux risques politiques.

Enfin, les grands groupes hôteliers ont souvent rencontré dans leur volonté d'expansion internationale des interlocuteurs empressés parmi les gouvernements, les collectivités. ou les investisseurs locaux des pays en voie de développement, favorisant encore ce phénomène d'internationalisation.

Ainsi, les pays d'Asie sont actuellement une zone privilégiée dans les stratégies de développement de nombreux groupes transnationaux au point que certaines localisations souffrent déjà d'une surcapacité, notamment sur le marché du haut de gamme, comme $c^{*} e s t$ le cas pour Singapour par exemple.

\section{Une segmentation des marchés plus fine}

Parallèlement au développement des chaines, celles-ci adoptent une approche de plus en plus répandue des marchés par la segmentation.
Ceci se traduit par deux types de stratégies:

- une stratégie de concentration sur un segment particulier, s'accompagnant de la définition plus pointue d'un produit hôtelier adapté aux attentes précises de la clientèle du segment visé. C'est une stratégie de marque. Exemples: en Amérique du Nord, Motel 6 dans le segment superéconomique ("economy"), Hyatt ou Four Seasons dans le segment haut de gamme ("super luxury").

- une stratégie de segmentation par le développement d'une véritable gamme de produits hốteliers contrôlés par le méme groupe, et permettant de couvrir différents segments du marché avec des marques diversifiées et adaptées (cf. tableau 2). Exemple: aux USA, des groupes comme Holiday Inns (avec les marques Hampton ou H.I. Crown Plaza) (cf. tableau 3), Quality International (Quality, Comfort, Clarion, Mc Sleep, et les produits "all suites" du groupe), en France Accor (Novotel, Mercure, Sofitel, Ibis, Urbis. Formule 1), ou Pullman International (Altea, Pull, PLM Azur, Arcade. Primo) (cf. tableau 1).

Les criteres de segmentation adoptés sont variables, les plus répandus étant les suivants:

- les prix ou la "catégorie" d"hỏtels (super economique, économique, standard, luxe. super luxe):

- la localisation (centre-ville, périphérie, aéroport, routes et autoroutes, stations touristiques. ...);
- le type de clientèle visé selon le motif du séjour (hommes d'affaires individuels, touristes individuels et groupes, congrès, ...):

Ces différents critères se recoupent ou se combinent pour aboutir à la définition d'un produit homogene.

Ainsi, on voit aujourd hui des produits stan= dardisés de chaines dans des créneaux du marché qui étaient auparavant réservés aux indépendants. C'est le cas en Europe, par exemple, des marchés économiques et super économiques. Un groupe comme Accord, a ainsi développé le concept certainement le plus avancé dans le domaine de l'hótellerie super économique: Formule 1 (ultrastandardisé, automatisé, sanitaires partagées entre quatre chambres, pas de restauration, ...) et son objectif avoué est d'ouvrir un millier d'établissements de ce type en Europe d'ici à la fin de la décennie 90 .

Certains observateurs ne manquent pas de dénoncer ce processus de segmentation poussée et la prolifération des enseignes qui en découle, saturant et encombrant ainsi certains marchés et déroutant le client. En outre, le développement de ces marques s'effectue trop souvent au détriment de franchisés ou d'investisseurs trop naifs. trompes par des études d'implantation trop sommaires.

\section{Une concentration économique et financière plus poussée}

La montée en puissance des chaines et l'émergence de grands groupes hốteliers s'est 


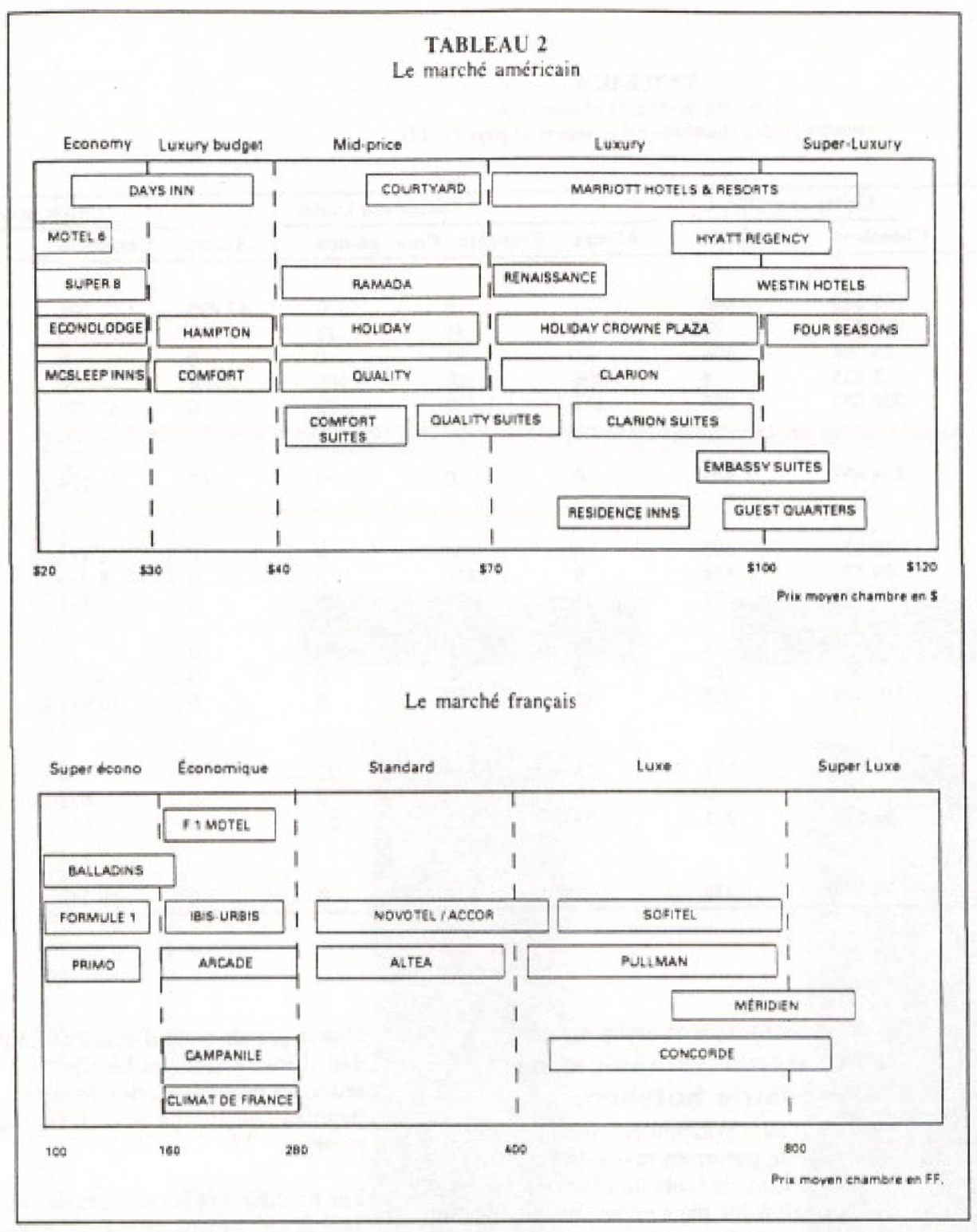

effectuée conjointement avec une concentration économique et financière du secteur. On a pu ainsi assister au cours des dernières années notamment, à de multiples fusions, absorptions, ventes et rachats de compagnies, encore accentuées par la mode des raiders.

Ceci trouve son origine d'une part dans la stratégie de croissance des grandes entreprises hôtelières, et d'autre part dans l'intérét porté à l'activité hôtelière par des groupes extérieurs au secteur.

Pour reprendre la théorie économique, on retrouve trois types d'integration économique:

- l'intégration verticale a été surtout le fait des compagnies aériennes, avec des exemples célèbres: Pan Am avec Intercontinental, TWA avec Hilton, Air France avec Méridien, Japan Airlines avec Nikko, et plus récemment United Airlines avec Westin et Hilton. Dans une moindre mesure, on peut citer également des compagnies ferroviaires: CP Hotels et CN Hotels développés par leurs entreprises ferroviaires respectives au Canada, ou Frantour par la SNCF en France.

Ceci n'est toutefois pas la tendance dominante aujourd 'hui: la plupart des compagnies aériennes américaines citées précédemment en proie à des difficultés dans leur secteur d'activité se sont désengagées de l'hôtellerie.

- la concentration horizontale est une ten$C^{\prime}$ est tout d'abord l'accroissement naturel des chaines qui aboutit, comme nous l'avons souligné, à une part de marché accrue. Mais c'est aussi un développement par fusions et absorptions, avec des exemples comme Trust House Forte. Ladbroke (récent acquéreur de Hilton International), Accor, Pullman (Compagnie Internationale des Wagons Lits et du Tourisme), qui se sont hissés en quelques années parmi les 20 premières entreprises hôtelières mondiales.

- La diversification est également un phédance plus répandue à l'heure actuelle. nomène en vogue ${ }_{4}$ car un certain nombre de grandes compagnies extérieures au secteur voient de bonnes potentialités d'investissement dans I'hôtellerie: c'est le cas en Grande-Bretagne, par exemple, de grands brasseurs (Grand Met, Bass) acquéreurs de sociétés hốtelières (Intercontinental, Holiday Inns International).

\section{La sophistication accrue du management}

L'arrivée des chaines a eu également pour effet d'apporter à l'hôtellerie - secteur économique en maturité, empreint de traditions - des techniques de gestion plus modernes, souvent empruntées à l'industrie. Ce processus s'est traduit à plusieurs niveaux, à savoir sommairement:

- la construction: la mise au point de produits hốteliers standardisés a permis une industrialisation de la construction autorisant des économies d'échelle sur les coûts des matériaux et des gains de temps sur les délais de construction. Un hôtel Formule 1 type, de 40 chambres est, par exemple, construit en 2 semaines.

Au niveau de la conception même du produit et de son implantation, des études préalables plus poussées sont fréquemment entreprises.

- la production en matière culinaire notamment est aujourd"hui facilitée, mieux maitrisée en termes de coúts, de qualité et de temps, grâce aux innovations technologiques intervenues dans les domaines des equipements de cuisine et des produits alimentaires (cuisson sous vide ou produits de 4 e gamme par exemple).

- La commercialisarion a evolué a la fois au plan conceptuel avec l'introduction des instruments du marketing, et au plan technique avec l'apparition des nouvelles technologies de communication. Ainsi, les techniques d'analyses du marché, de promotion, de publicité, de planification et autres outils du marketing se sont imposés dans l'hôtellerie. L'hótelier n'attend plus le client, il va le chercher sur des marchés parfois lointains, met en place des réseaux de vente, utilise des systèmes informatisés internationaux de réservation. La télématique le met en contact direct et en temps réel avec ses clients et va modifier profondément les réseaux de distribution.

- la gestion des établissements a connu aussi sous l'impulsion des chaines l'introduction de techniques de management auparavant réservées à d'autres secteurs et ceci dans divers domaines, outre le marketing déjà cité: gestion comptable et financière, gestion des équipements, ressources humaines, ...

Les chaines ont harmonisé les procédures de gestion de leurs unités et joué dans ce domaine un róle de formation important. 
TABLEAU 3

Les cinq premières chaines US:

répartition des chambres selon statuts et pays (1987)

\begin{tabular}{|c|c|c|c|c|c|c|c|c|}
\hline \multirow[t]{2}{*}{ Noms } & \multicolumn{2}{|c|}{ Unités aux USA } & \multicolumn{4}{|c|}{ Statuts des Unités } & \multicolumn{2}{|c|}{ Unités hors USA } \\
\hline & Chambres & Hotels & Filiales & Franchises C & tion & Autres & Chambres & Hótels \\
\hline \multicolumn{9}{|l|}{ Holiday Corporation } \\
\hline Holiday Inn \& Crown Plazas & 269543 & 1440 & 1294 & 0 & 0 & 47495 & 201 & \\
\hline Embassy Suites & 17254 & 73 & 20 & 31 & 22 & 0 & 0 & 0 \\
\hline Hampton Inn & 13394 & 106 & 11 & 95 & 0 & 0 & 0 & 0 \\
\hline Harrah's & 2853 & 4 & NR & NR & NR & NR & 0 & 0 \\
\hline TOTAL & 303044 & 1623 & 117 & 1420 & 22 & 0 & 47495 & 201 \\
\hline \multicolumn{9}{|l|}{ Best Western International } \\
\hline Best Westerns & 174059 & 1905 & 0 & 0 & 0 & 1905 & 81554 & 1441 \\
\hline \multicolumn{9}{|l|}{ Quality International } \\
\hline Comfort Inns & 36.921 & 400 & 0 & 400 & 0 & 0 & 1197 & 19 \\
\hline Quality Inns & 60571 & 478 & 9 & 466 & 3 & 0 & 8734 & 76 \\
\hline Clarion / Hotels & 10394 & 32 & 1 & 28 & 3 & 0 & 511 & 5 \\
\hline \multicolumn{9}{|l|}{ Inns / Resorts } \\
\hline Comfort Suites & 1231 & 8 & 0 & 8 & 0 & 0 & 0 & 0 \\
\hline Quality Suites & 1561 & 10 & 0 & 10 & 0 & 0 & 0 & 0 \\
\hline TOTAL & 110678 & 928 & 10 & 912 & 6 & 0 & 10442 & 100 \\
\hline \multicolumn{9}{|l|}{ Hilton Hotels Corporation } \\
\hline Hilton Hotels & 97535 & 271 & 25 & 223 & 22 & 0 & 0 & 0 \\
\hline Conrad International Hotels & 0 & 0 & 0 & 0 & 0 & 0 & 622 & 1 \\
\hline TOTAL & 97535 & 271 & 25 & 223 & 22 & 0 & 622 & 1 \\
\hline \multicolumn{9}{|l|}{ The Sheration Corporation } \\
\hline Sheraton Hoiels & 95828 & 379 & 57 & 322 & 0 & 0 & 48342 & 127 \\
\hline
\end{tabular}

Source: Lodging Hospitality / August 1987

L'informatisation croissante des hótels (même si elle reste encore limitée: 800 établissements sur 18000 en France) apporte un appui pour bon nombre de fonctions: réservations (avec gestion des groupes, des contingents, des sur-réservations, ...), réception (arrivées, départs, statistiques d'occupation, fichiers clients, ...), comptabilité, paye, facturation automatique permanente, caisse, gouvernante, ...

Les institutions de formation ont aussi une part importante dans cette évolution vers une technicité accrue en management: les matières correspondantes ont fait leur apparition dans les programmes des formations hôtelieres, et en complément des écoles hớtelières traditionnelles assurant la formation aux techniques professionnelles sont apparues des institutions universitaires proposant l'apprentissage des techniques de management appliquées au secteur hôtelier.

\section{Les dimensions économiques de I'activité hôtelière}

Face à ces évolutions structurelles des marchés, comment ont évolué les performances économiques et la rentabilité de ce qu'il est convenu désormais d'appeler l'industrie hótelière - mëme si le terme d'industrie sied assez mal à cette activité?

Nous allons, avant de tenter de répondre à cette question, examiner les montages financiers et le financement en vigueur dans ce secteur.

\section{Les financements et les montages financiers de I'industrie hôtelière}

Il n'est pas, évidemment, dans l'objet de cet article de passer en revue de détail la panoplie des instruments de financement, variables selon les pays et les époques (2). Nous pouvons en donner toutefois un aperçu général sommaire.

L'hôtellerie est une activité de main-d"oeuvre, mais en meme temps, une industrie à haute intensité capitalistique par le volume des investissements requis au niveau de l'immobilier principalement et des equipements. Schématiquement, comme dans certaines industries lourdes, il faut 3 Fr de capital pour obtenir 1 Fr de chiffre d'affaires ${ }^{04}$. Dans les pays développés, les coûts d'investissements peuvent varier de $100000 \mathrm{FF}$ par chambre (exemple pour un hôtel Formule 1) a $1000000 \mathrm{FF}$ (pour du haut de gamme) selon le type d'hôtel et sa localisation. La question du financement est donc fondamentale dans ce secteur.

Durant les 10 dernières années le financement de l'hótellerie a connu beaucoup de changements en raison bien sûr des modifications de structures et d'échelle de ce secteur, mais aussi à cause d'une incertitude économique grandissante, du flux puis du reflux de l"inflation et des taux d'intérët. Tout ceci a affecté l'invest issement hôtelier de manière significative.
Quatre grands types d'acteurs sont impliqués dans l'investissement hótelier: les investisseurs, les promoteurs-développeurs, les compagnies gestionnaires, les organismes prểeurs.

Les méthodes et les sources de financement varient en fonction de la conjoncture économique: ainsi en période d'expansion inflationniste (années 70), 1 'investissement immobilier esı considéré comme une bonne solution face à l'inflation; les compagnies d'assurance, les fonds de pension dirigent une plus grande proportion de leurs fonds des valeurs mobilières vers l'immobilier. Inversement, en période de récession (début des années 80), et lorsque l'inflation décroit pendant que les taux d'intérêt restent élevés, 1 'investissement immobilier n'est plus privilégié. L'investissement hôtelier est liế au marché immobilier et s'avère donc sensible aux facteurs précédemment décrits.

Plus spécifiquement et sommairement, les chaines intégrées recourent généralement aux méthodes suivantes de financement de leur développement:

- investissements directs: en fonds propres par autofinancement ou par appel public à l'epargne (augmentation de capital ev/ou appel au marché financier). La chaine est alors propriétaire de I'immobilier.

- contrat de franchise: la chaine vend son enseigne et son savoir-faire à un investisseur-exploitant, et lui apporte une aide dans la gestion et la commercialisa- 
TABLEAU 4

Les vingt premiers groupes hôteliers mondiaux

\begin{tabular}{|c|c|c|c|}
\hline Rang & Firme & $\begin{array}{c}\begin{array}{l}\text { Nombre de } \\
\text { chambres } \mathbf{8 7}\end{array} \\
\end{array}$ & $\begin{array}{c}\text { Nombre } \\
\text { d'unitis } 87\end{array}$ \\
\hline $1^{2}$ & Holiday Corporation. USA & 352893 & 1832 \\
\hline 2 & Sheration Corporation, USA & 136495 & 479 \\
\hline $3^{5}$ & Ramada Inc., USA & 123915 & 742 \\
\hline 4. & Marioul Corporation, USA & 10300 & 361 \\
\hline 5 & Quality International, USA & 102428 & 878 \\
\hline $6 \%$ & Hilton Hotels Corporation, USA & 97000 & 272 \\
\hline $7 z^{2}$ & Days Inn of America Ine., USA & 84832 & 590 \\
\hline 7 & Accor, France & 84800 & 713 \\
\hline 9 & Trusthouse Fone PLC, Grande Bretagne & 84371 & 809 \\
\hline 10 & Prime Motor Inns, USA & 74006 & 586 \\
\hline 11. & Balkantourist, Bulgarie & 56250 & 386 \\
\hline 12 & Club Méditerranée, France & 55152 & 213 \\
\hline 13 & Bass Hotel Division, Grande Bretagne & 52436 & 293 \\
\hline 14 * & Motel 6, USA & 48750 & 431 \\
\hline 15 & Hyatt Hotels Corporation, USA & 48427 & 89 \\
\hline 16 & Sol Hotels, Espagne & 45000 & 139 \\
\hline 17 & Ladbroke Group PLC, Grande Bretagne & 44127 & 145 \\
\hline 18 & Grand Metropolitain PLC, Grande Bretagne & 38725 & 157 \\
\hline 19. & Radisson Hotel Corporation, USA & 37500 & 158 \\
\hline 20 & Westin Hotel \& Resorts, USA & 34291 & 62 \\
\hline
\end{tabular}

Source: Hotels $\boldsymbol{\alpha}$ Restaurants International

tion de son établissement moyennant un droit d'entrée et une redevance (en pourcentage du CA).

- contrat de gestion: la chaine gère l'ćtablissement pour le compte d'un propriétaire investisseur qui perçoit les bénéfices. La compagnie gestionnaire est rémunérée par une redevance (combinaison négociée d'un pourcentage sur le CA et sur le revenu brut d'exploitation). Elle peut être amenée à participer pour un montant plus ou moins important au financement de l'investissement avec prise de participation au capital.

- constitution de fonds communs de placement à risques, créés à partir de l'épargne publique et de capitaux d'investisseurs institutionnels (assurances, fonds de pension, ....), avec qui la chaine passe des contrats de location.

Il va de soi que, dans ces montages, la propriété de l'immobilier est le plus généralement séparée juridiquement de l'exploitation.

Ces modes de financement n'excluent pas. bien entendu, le recours aux institutions de crédit (banques et établissements financiers) pour des prèts à long et moyen termes aux conditions du marché.

Il faut mentionner également la formule du crédit-bail (leasing), dans laquelle l'exploitant fait acquérir les biens immobiliers par une société financière spécialisće qui les lui $70 \%$ du total des préts a l'hotellerie. sa santé.

\section{Les risques et les profits de I'investissement hôtelier} equipements.
$20 \%$ du total de l'investissement.

Les spécificités de l'investissement hớtelier tiennent à trois éléments essentiels ${ }^{(t)}$ :

- les équipements incluant les chambres, les salons et salles à manger, les cuisines, la réception et les équipements administratifs, comptent jusqu'à $25 \%$ dans l'investissement.

- l'hötellerie est un commerce de détail avec un turn-over important de clientèle - requérant une main-d 'oeuvre qualifiée et un encadrement spécialisé. C'est une activité de main-d'oeuvre et la masse salariale peut représenter jusqu'à $40 \%$ du CA selon le type d'établissement.

- I'hôtellerie requiert des stocks et un fonds de roulement conséquents.

Ces caractéristiques vont avoir une influence sur les risques et les benéfices de cette activité. Mais pour évaluer la rentabilité de ce type d'investissement, il convient de prendre également en considération les facteurs suivants:

- une période de démarrage plus ou moins longue avant d'atteindre une vitesse de croisière et une profitabilité adéquate. Il faut généralement prévoir 1 à 4 années de cash-flows négatifs.

- l'activité de restauration comporte des risques élevés et des perspectives de profits limitées. Il s'agit toutefois d"un service à la clientẻle nécessaire pour des raisons de concurrence. Mis à part des établissements ayant un gros volume de banquets. ce département est généralement déficitaire apress une affectation precise des coûts fonctionnels.

Enfin, certains pays ont mis en place des systèmes de prêts bonifiés afin de favoriser l'investissement hôtelier de manière générale ou sélective, particulièrement destinés à telle ou telle catégorie d'hôtels. En France, ces prêts passent par l'intermédiaire du CEPME (ex: Crédit Hôtelier) qui représente environ

Les modes de développement privilégiés par de nombreuses chaînes sont la franchise et le contrat de gestion. Ceci ne manque pas d'avoir une influence sur les structures de l'industrie hởtelière, particulièrement aux USA: par ce biais, les sociétés hôtelières ne contrölent plus vraiment l'industrie car elles ne sont plus propriétaires des unités. Elles deviennent ainsi de plus en plus des fournisseurs de services, et certains observateurs dénoncent le risque de les voir davantage intéressés par la croissance de l'offre que par

Les hôtels sont un investissement immobilier d'un type particulier et hautement spécialisé. Ils consistent en terrain, bătiments et

Le terrain représente généralement moins de
- I'obsolescence relativement rapide des décors et equipements, encore accentuée par la prolifération de nouveaux concepts. et établissements, est un facteur d'érosion des profits. Ainsi, un hớtelier est en perpétuel réinvestissement dans l'un ou l'autre de ses départements, s'il ne veut pas se trouver finalement devant une masse de rénovations impossible à supporter.

- les risques liés à l'environnement macroéconomique et commercial. Des facteurs externes peuvent affecter gravement la demande hôtelière, principalement dans les segments touristiques (crise economique, changements dans les voies de communications, dans les centres d'intérét urbains, etc...).

Toutefois, il y a malgré ces risques, un potentiel pour des profits intéressants. Lorsque le seuil de rentabilité est atteint, les profits tendent à croittre rapidement: une large part $(80 \%$ a $90 \%)$ des charges est fixe et les dépenses ne varient donc pas de façon significative avec l'occupation.

Les retours sur investissements sont dérivés du cash-flow annuel après service de la dette (rémunération du capital), des rembourse- 


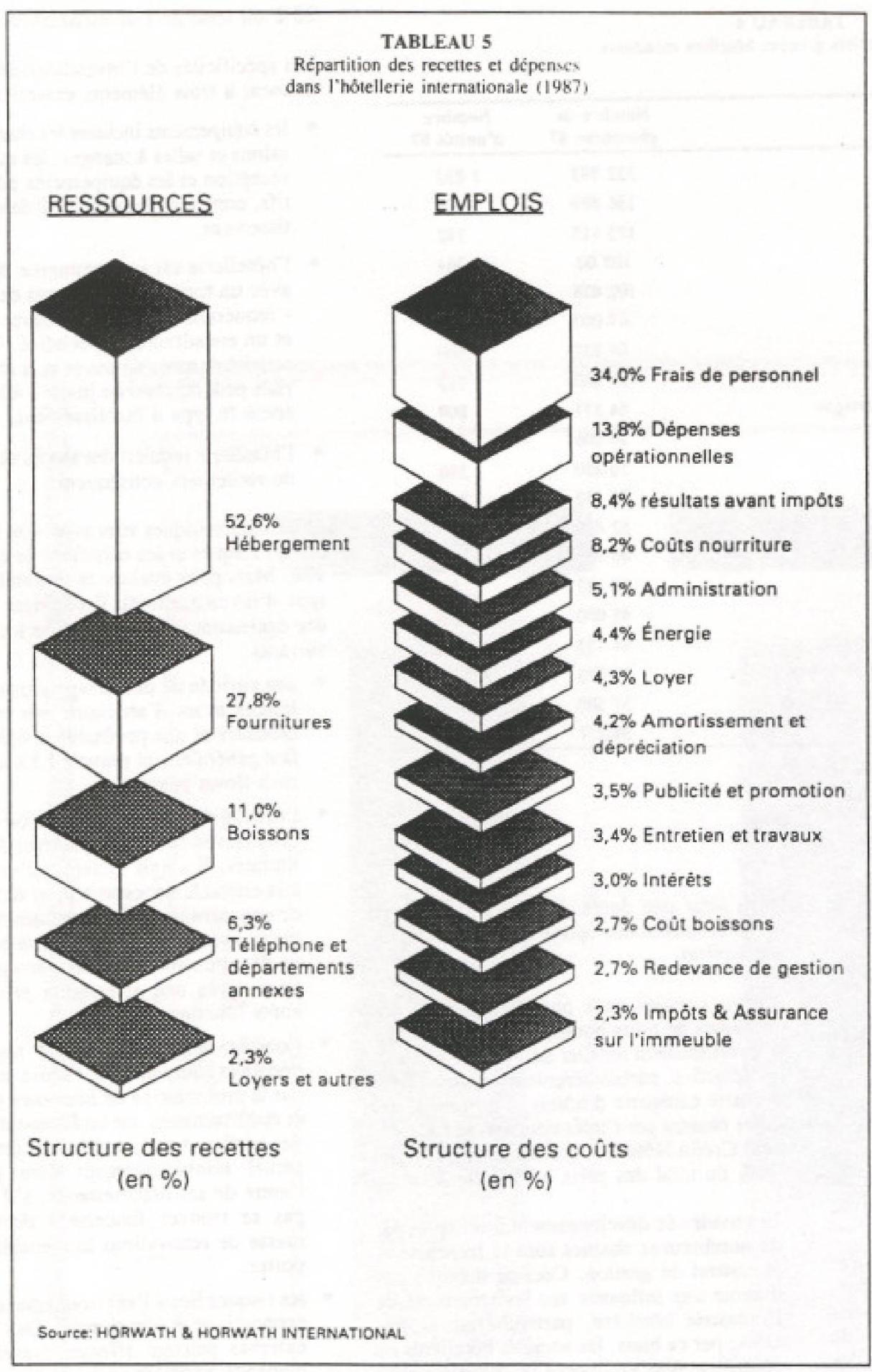

ments d'emprunts hypothécaires, et de la plus-value potentielle réalisée en cas de vente de l'établissement. La rentabilité finale dépend d"une variété de facteurs incluant les conditions générales du marché, l'évolution des facteurs de risques liés à l'hötellerie en général et à la localisation particulière d'un établissement, les ratios d'offre et de demande, la disponibilité et le coût des financements, et les avantages fiscaux.

Lés investisseurs hôteliers, au cours des années 70 à 85 environ, ont recherché une rentabilité des capitaux propres variant entre des maxima de $20 \%$ à $25 \%$ jusqu'à des minima de $8 \%$ a $12 \%$. supérieurs avec des taux d'occupation moindres.

Le tableau 5 nous montre la structure des ressources et des emplois (répartis selon le Uniform System of Accounts for Hotels). a l'échelle internationale. Les moyennes présentées font ressortir, comme nous avons pu le mentionner précédemment. la part prépondérante des charges fixes, les dépenses liées directement au volume des ventes n'excédant pas $20 \%$ du total en général, avec un poids important pour les coùts salariaux: I'hơtellerie est une activité requérant des investissements lourds, mais c'est en mểme temps une activité de main-d'oeuvre.

Dans ce contexte, les résultats bruts d'exploitation oscillent généralement entre $20 \%$ et $35 \%$ du CA, selon la catégorie d'hơtel (les catégories supérieures ayant des RBE souvent plus faibles en raison précisément des services à fournir aux clients et des coutts de personnel qui en découlent. Les résultats avant impóts $-8 \%$ à $10 \%$ en moyenne internationale - oscillent entre $0 \%$ et $15 \%$ selon la zone géographique.

Concernant l'évolution des performances, on peut constater qu'après une année 1986 généralement mauvaise (exception faite de l'Amérique du Nord), l'année 1987 a marqué une remontée des résultats (la zone nordaméricaine encore mise à part). Les performances hôtelières sur ce continent nordaméricain traduisent un déséquilibre croissant offre-demande, avec la mise sur le marché d'un nombre important de nouveaux établissements qui n'ont pu être absorbés par une demande en relative stagnation. Mais le mème constat peut être fait à un degré moindre pour d'autres zones géographiques.

Il est difficile d'atteindre des résultats d'exploitation suffisants pour dégager un résultat net intéressant au plan de la rentabilité. Cela tient à plusieurs facteurs: d'une part la hausse des coûts (notamment salariaux, poste principal des dépenses), et d'autre part, la concurrence accrue, face à une demande croissant moins vite que l'offre. Ceci empéche une répercussion totale des hausses de coûts sur les prix de vente, ce double phénomène pesant donc sur les profits.

Au terme de ces pages, on s'aperçoit donc que l'hôtellerie est une industrie lourde et de main-d'oeuvre, même si elle est encore dominée par de petites entreprises familia. les, en dépit de la croissance des chaines. Ces caractéristiques lui donnent une relative rigidité, qui l'handicape face à l'apparition de nouveaux modes d'hébergement, notamment sur le segment touristique, et face à sa propre croissance. Il s"agit également pour ce secteur d'activité de bien maîtriser son évolution. Cela signifie entre autres choses, de négocier correctement le passage au stade véritablement industriel dans ses structures et ses modes de fonctionnement et de ges. tion, sans pour autant tourner le dos aux valeurs de service et d'accueil qui font son essence même. $f$ 
TABLEAU 6

Performances de l'hớtellerie internationale par zone géographique

\begin{tabular}{|c|c|c|c|c|c|c|c|c|c|c|c|c|}
\hline \multirow[t]{2}{*}{ Statistiques générales } & \multicolumn{2}{|c|}{ Tous les hötels } & \multicolumn{2}{|c|}{$\begin{array}{c}\text { Afrique et } \\
\text { Moyen-Orient }\end{array}$} & \multicolumn{2}{|c|}{$\begin{array}{l}\text { Asie et } \\
\text { Australie }\end{array}$} & \multicolumn{2}{|c|}{ Amérique du Nord } & \multicolumn{2}{|c|}{ Europe } & \multicolumn{2}{|c|}{$\begin{array}{c}\text { Amérique latine/ } \\
\text { Caralbes }\end{array}$} \\
\hline & 1987 & 1986 & 1987 & 1986 & 1987 & 1986 & 1987 & 1986 & 1987 & 1986 & 1987 & 1986 \\
\hline Taux d"occupation & $66.4 \%$ & $64.5 \%$ & $53.0 \%$ & $55.9 \%$ & $76.4 \%$ & $67.9 \%$ & $66.5 \%$ & $68.5 \%$ & $64.9 \%$ & $62.8 \%$ & $68.8 \%$ & $63.4 \%$ \\
\hline Taux de double occupation & 39.9 & 36.2 & 21.6 & 21.8 & 50.0 & 45.6 & 43.9 & 46.6 & 34.2 & 31.6 & B5.8 & 70.4 \\
\hline Prix Moyen (\$) & & & & $=$ & & & & & & & & \\
\hline - par chambre & 62.13 & 56.72 & 65.89 & 58.72 & 55.39 & 51.65 & 61.45 & 61.66 & 64.72 & 56.53 & 42.68 & 55.57 \\
\hline - par client & 49.5 & 43.79 & 55.46 & 50.23 & 38.14 & 36.15 & 52.74 & 43.73 & 51.58 & 45.05 & 28.99 & 39.43 \\
\hline \multicolumn{13}{|l|}{$\begin{array}{l}\text { Revenu brut d'exploitation } \\
\text { (RBE) (\%) }\end{array}$} \\
\hline - ratio sur CA hébergement & 49.7 & 43.4 & 47.6 & 43.6 & 56.0 & 45.4 & 37.9 & 38.3 & 54.5 & 48.3 & 47.9 & 44.5 \\
\hline - ratio súr CA total & 25.6 & 22.9 & 20.6 & 18.9 & 27.6 & 20.3 & 21.7 & 23.2 & 27,4 & 24.8 & 28.7 & 25.1 \\
\hline Rotation clients & 121 & 107 & 104 & 89 & 177 & 108 & 97 & 100 & 128 & 114 & 124 & 118 \\
\hline \multicolumn{13}{|l|}{ Répartition des ventes $\left(\varphi^{\prime}\right)^{*}$} \\
\hline - hébergement & 53,6 & 56.0 & 45.2 & 53.1 & 51.7 & 50.8 & 63.6 & 51.8 & 52.2 & 55.5 & 53.1 & \\
\hline - nourriture & 26.4 & 24.6 & 28.1 & 21.7 & 27.2 & 27.7 & 23.8 & 22.1 & 27.8 & 27.4 & 23.0 & 24,6 \\
\hline - boissons & 11.6 & 10.7 & 11.0 & 8.3 & 10.1 & 10.3 & 9.5 & 8.7 & 13.5 & 13.8 & 11.4 & 10.5 \\
\hline - ıéléphone & 2.4 & 2.2 & 4.7 & 3.5 & 4.1 & 4.1 & 1.6 & 1.5 & 1.6 & 1.5 & 2.9 & 3.1 \\
\hline - départements annexes & 2.4 & 2.2 & 4.7 & 3.5 & 4.1 & 4.1 & 1.6 & 1.5 & 1.6 & 1.5 & 2.9 & 3.1 \\
\hline - loyers / revenus accessoires & 2,4 & 2.8 & 3.2 & 6.3 & 3.3 & 3.3 & 1.5 & 1.6 & 2.1 & 1.7 & 3.2 & 4.6 \\
\hline TOTAL & 100.0 & 100.0 & 100.0 & 100.0 & 100.0 & 100.0 & 100.0 & 100.0 & 100.0 & 100.0 & 100.0 & 100.0 \\
\hline \multicolumn{13}{|l|}{ Indicateurs par chambre (5) } \\
\hline - CA total & 28,290 & 24,329 & 24,983 & 24.102 & 31.150 & 26,709 & 24,456 & 23,963 & 31,020 & 25,844 & 20,518 & 25,582 \\
\hline - R B E & 7,314 & 5.790 & 5,957 & 4.379 & 9,638 & 5,952 & 5,409 & 5,398 & 9,099 & 6.811 & 4.486 & 5,690 \\
\hline - Resultar avant impót & 2,834 & 1,626 & 2,704 & 1.874 & 5,674 & 2.142 & $(307)$ & 676 & 4,563 & 2,553 & 2.038 & 2.452 \\
\hline
\end{tabular}

* Tous ces autres chiffres sont des médianes

Source: Horwath \& Horwath International

TABLEAU 7

Evolution comparée des performances hótelières (en \%)

\begin{tabular}{lccccc}
\hline & 1987 & $\$ 986$ & 1985 & 1984 & 1983 \\
\cline { 2 - 5 } $\begin{array}{l}\text { International } \\
\text { - Taux d'occupation(1) }\end{array}$ & 66.4 & 64.5 & 67.1 & 67 & 64.6 \\
- R B E & & & & & \\
- & 25.6 & 22.9 & 23.9 & 22.3 & 23.8 \\
Europe & & & & & \\
- Taux d'occupation & 64.9 & 62.8 & 67.1 & 66.6 & 65.4 \\
- R B E & 27.4 & 24.8 & 23.5 & 26.3 & 24.6 \\
Amérique du Nord & & & & & \\
- Taux d'occupation & & 68.5 & 67.0 & 68.1 & 62.9 \\
- R B E & 66.5 & 23.2 & 22.9 & 20.5 & 20.6 \\
\hline
\end{tabular}

(1) Taux d'occupation en of des chambres

(2) R B E en $\%$ du CA total

(3) La zone Amérique du Nord comprend:

USA + Canada en 1987 et 1986

USA + Canada + Caraibes en 83,84 et 85

Source: Horwarth \& Horwarth International
Notes explicatives

(1) Citee par Horwath et Horwath International dans Worldwide Hotel Industry 7987, p. 7.

(2) Cf. James Evster ICornell University), Finaneing the Lodging Industry - Players and Techniques of the 80 's, Laventhol if Horwath, 1982.

(3) Rapport au Constil Eeonomique et Social, Les conditions d'un nouva au developpement de l'hótelle. rie, Paris, 1987

(4) S. Rushmore, Hotals. Motels and Restaurants Valuation and Market Studies. American Institute of Real Estate Appraisers, 1983.

\section{Bibliographie compldmentaire}

Glenn WITHIAM, Hotel Companies Aim for Multiple Markets, Comell Ouarterly, November 1985.

Lodging Hospitality - Lodging's 400 top Performers (August 1987).

PANNEL, KERR, FORSTER, Trends in the Hotel Industry (édition annuelle).

HORWATH ET HORWATH, France. L'industrie hotelière française (édition annuellel.

Revue Technique des Hótels et Restaurants, Les chaines hótelieres françaises liuillet 1989).

Quality International, Revue Portfolio (printemps 1968). 